\title{
Traumatic humeral articular cartilage shear (THACS) lesion in a professional rugby player: a case report
}

\author{
I-H Jeon, W A Wallace
}

Br J Sports Med 2004;38:e12 (http://www.bjsportmed.com/cgi/content/full/38/4/e12). doi: 10.1136/bjsm.2003.007708

\begin{abstract}
A 20 year old male professional rugby player was seen at the clinic for evaluation of shoulder pain after rugby play. Magnetic resonance imaging showed extensive subchondral bone bruising of the humeral head with defect of the articular cartilage. Arthroscopy showed that the inferior half of the humeral head had extensive articular cartilage loss with nearly $70 \%$ of the inferior head having lost its cartilage. Sports medicine doctors should be aware that the shoulder joint in young competitive athletes playing contact sports may be exposed to greater risk of this kind of injury.
\end{abstract}

$\mathrm{R}$ ugby Union at a professional level is an aggressive contact sport with a high risk of shoulder injury. Although chondral or subchondral lesions are recognised as a source of pain in the knee, however, there has been no description in the literature of extensive articular cartilage detachment of the humeral head resulting from an injury at rugby, as was the case with this patient.

\section{CASE REPORT}

A 20 year old male professional rugby player was referred to us for evaluation of shoulder pain 8 days after suffering an injury while playing rugby. He was a winger, playing in the backs for a Premiership Rugby Union team. He reported no shoulder problems prior to this injury. However, his shoulder pain developed after he fell heavily to the ground onto his left shoulder during the match and another player fell on top of him causing severe pain in the left shoulder although he did not feel that his shoulder was dislocated. Despite severe pain in his shoulder, he continued playing for $15 \mathrm{~min}$, but then came off the field because of the pain.

When assessed 7 days later, he had a sensation of clicking from the left shoulder, which did "not feel right". Examination of his left shoulder revealed active forward flexion to $150^{\circ}$, abduction to $90^{\circ}$, and an obvious subacromial effusion. The apprehension test for anterior instability (external rotation at $90^{\circ}$ abduction) was positive. He had good strength of both supraspinatus and infraspinatus muscles.

Plain radiographs revealed a normal glenohumeral articulation with no visible impression fracture or bony Bankart lesion. Magnetic resonance imaging (MRI) identified an articular cartilage lesion of the humeral head showing extensive subchondral bone bruising of the humeral head. There was apparent cartilage thinning and a defect of the articular cartilage of the head with irregularity at the dome (fig 1).

Examination under anaesthesia 3 weeks after injury revealed grade II anterior laxity with grating and diagnostic arthroscopy showed that the inferior half of the humeral head (below the equator) had extensive articular cartilage detachment with sharp edges involving nearly $70 \%$ of the
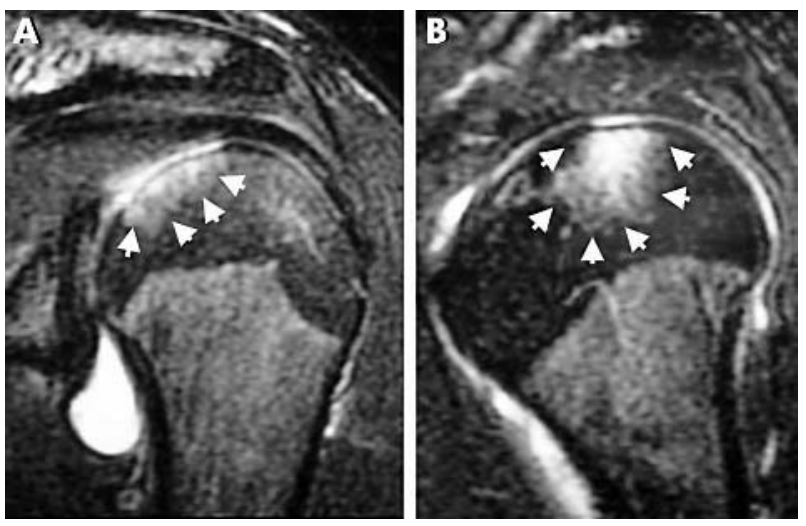

Figure 1 (A) Coronal and (B) oblique sagittal fast spin echo T2weighted MR image with fat suppression in the left shoulder reveals articular cartilage defect (arrows) of humeral head and diffuse subchondral bone bruise along the posterosuperior head. Anterior is to the left. Defects with subjacent marrow oedema are located at the dome of the head apart from a typical Hill Sachs lesion.

inferior half of the humeral head. The cartilage posteroinferiorly was spared but the remaining inferior humeral head was essentially exposed subchondral bone only (fig 2). A small number of loose bodies (articular cartilage flakes) in the shoulder were removed. With the arm by the side in $30^{\circ}$ internal rotation, the humeral head was essentially subchondral bone articulating with the glenoid cartilage.

\section{DISCUSSION}

Rugby is a highly competitive contact or collision sport and injuries to the shoulder are common. Although upper limb injuries account for only $15.4 \%$ of all injuries, $55.6 \%$ of all shoulder injuries have been identified as being severe. ${ }^{1}$ At the elite level, an increase in shoulder girdle injuries related to rotator cuff tear and instability have been reported. ${ }^{2}$ However there has been no report in the literature of articular cartilage detachment of the humeral head injured during rugby.

Shoulder MR imaging in general is particularly important in helping identify abnormalities that may mimic rotator cuff or labral abnormalities at clinical examination, avoiding unnecessary surgery; the accuracy of articular cartilage assessment with MRI has also greatly improved recently. In this case, preoperative MRI also demonstrated diffuse chondral irregularity and defect and thinning of the superior humeral head with subchondral oedema.

Osteochondritis dissecans ${ }^{3}{ }^{4}$ of the humeral head has been reported and focal cartilage defects of the articular surface of the superior humeral head in MRI have been reviewed by

Abbreviations: MRI, magnetic resonance imaging; THACS, traumatic humeral articular cartilage shear 


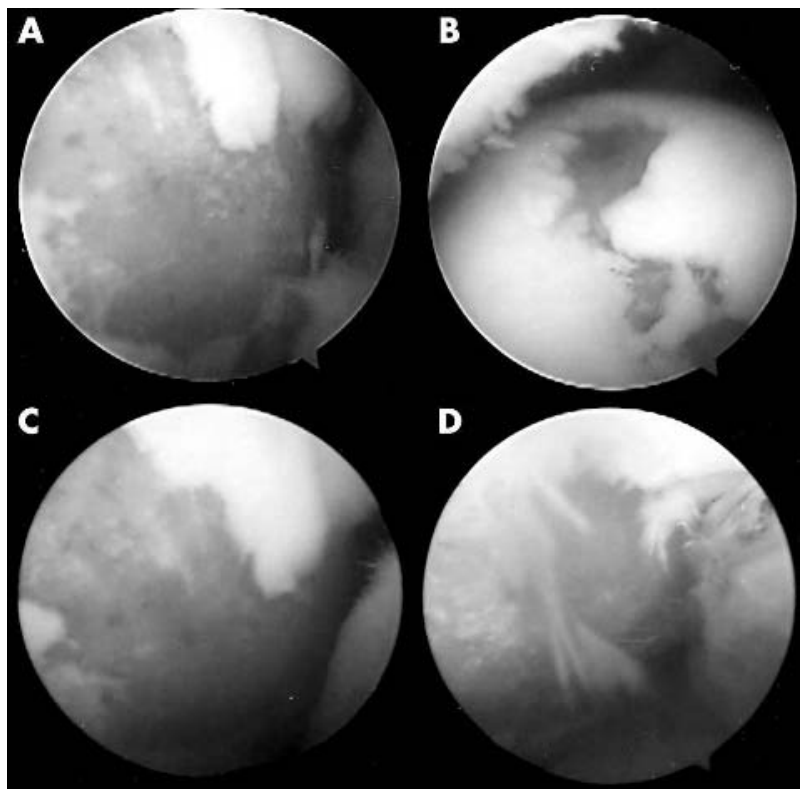

Figure 2 Arthroscopic picture looking along the humeral head confirms the articular cartilage defect with the underlying subchondral bone confirmed at arthroscopy. (A) Anterosuperior part of the head; (B) posterosuperior part of the head; $(C, D)$ inferior hemisphere of the head presents extensive articular cartilage shear fracture.

Carroll et al, ${ }^{5}$ the largest one in their series being $2 \times 3 \mathrm{~cm} .{ }^{5}$ In this case, arthroscopy revealed a traumatic massive articular cartilage detachment with a sharp edge involving more than $70 \%$ of the humeral head (fig 2).

Articular surface injuries are influenced by type of loads and rate of loading. ${ }^{67}$ Twisting and spraining injuries and joint dislocations subject the convex articular surface to high shear forces, which in turn may induce lesions in the cartilage and bone. In this case, a sudden subluxation or perhaps dislocation was considered to be the cause of the injury.

In summary, there should be increasing awareness that the shoulder joint in young competitive athletes undergoing contact or collision sports may be exposed to a greater risk of this kind of injury. Thus, sports medicine doctors who treat the shoulders of high demand athletes should be aware of the traumatic humeral articular cartilage shear (THACS) lesion. Preoperative MR imaging can provide valuable information

\section{Take home message}

Sports medicine doctors should be aware that the shoulder joint in young competitive athletes playing contact sports may be exposed to greater risk of traumatic humeral articular cartilage shear (THACS) lesion.

to the surgeon who should then inspect this lesion with arthroscopy. The management of this lesion is currently problematic as there is no effective treatment currently available. This patient was allowed to return to full competitive rugby as the authors felt that there was no effective treatment currently available and the medium term prognosis was unlikely to be worsened by using the shoulder for sport.

\section{Authors' affiliations}

I-H Jeon, W A Wallace, Nottingham Shoulder and Elbow Unit, Nottingham City Hospital, Nottingham, UK

The authors have not and will not receive any financial benefit in association with the present paper.

Conflict of interest: none declared.

Correspondence to: In-Ho Jeon, Nottingham Shoulder and Elbow Unit, Charnley Suite, Nottingham City Hospital, Hucknall Road, Nottingham, NG5 1PB, UK; jeonchoi@chol.com

Received 9 August 2003

Accepted for publication 11 August 2003

\section{REFERENCES}

1 Bathgate A, Best JP, Craig G, et al. A prospective study of injuries to elite Australian rugby union players. Br J Sports Med 2002;36:265-9.

2 Best JP, Robinson B, Myers P. Rotator cuff tears in elite Australian rugby players. In: Conference Handbook, Australian College of Sports Physicians Annual Conference. Brisbane: Ozaccom, 2000:16-25.

3 Hamada S, Hamada M, Nishive S, et al. Osteochondritis dissecans of the humeral head. Arthroscopy 1992;8:132-7.

4 Johnson DL, Warner JJ. Osteochondritis dissecans of the humeral head: treatment with a matched osteochondral allograft. J Shoulder Elbow Surg 1997;6:160-3

5 Carroll KW, Helms CA, Speer KP. Focal articular cartilage lesions of the superior humeral head: MR imaging findings in seven patients. Am J Roentgenol 2001;176:393-7.

6 Buckwalter JA. Articular cartilage injuries. Clin Orthop 2002;402:21-37.

7 Flachsmann RM, Broom ND, Hardy AE, et al. Why is the adolescent joint particularly susceptible to osteochondral shear fracture? Clin Orthop 2000;381:212-21. 